\title{
LA-UR-97- 413
}
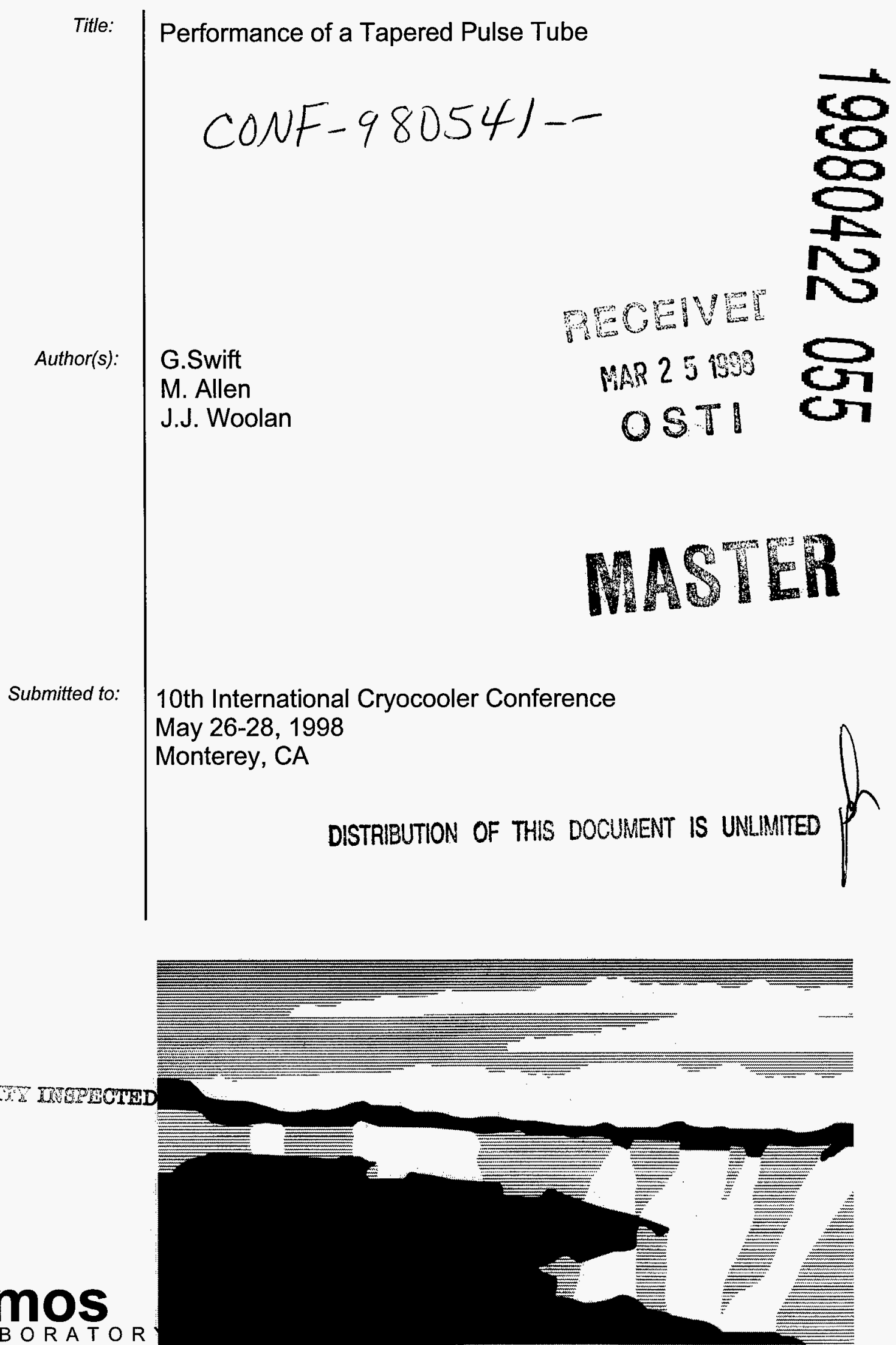


\section{DISCLAIMER}

This report was prepared as an account of work sponsored by an agency of the United States Government. Neither the United States Government nor any agency thereof, nor any of their employees, makes any warranty, express or implied, or assumes any legal liability or responsibility for the accuracy, completeness, or usefulness of any information, apparatus, product, or process disclosed, or represents that its use would not infringe privately owned rights. Reference berein to any specific commercial product, process, or service by trade name, trademark, manufacturer, or otherwise does not necessarily constitute or imply its endorsement, recommendation, or favoring by the United States Government or any agency thereof. The views and opinions of authors expressed herein do not necessarily state or reflect those of the United States Government or.any agency thereof. 


\section{Performance of a tapered pulse tube \\ G. W. Swift, ${ }^{1}$ M. ?. Allen, and J. J. Wollan \\ Cryenco Inc., 3811 Joliet, Denver CO 80239}

To be presented at ICC-10

\section{Abstract}

In a well instrumented pulse tube refrigerator having $1500 \mathrm{~W}$ of cooling power at $125 \mathrm{~K}$, we have measured the figure of merit of a tapered pulse tube at several operating points. At operating points near the operating point for which the taper was designed (using the design method described by Olson and Swift, to be published in Cryogenics in late 1997), the figure of merit is 0.96. This is close to the theoretical optimum figure of merit 0.97 calculated for this pulse tube considering only two loss mechanisms: heat conduction in the metal pulse tube wall and ordinary thermoacoustic heat transport in the gas within a few thermal penetration depths of the wall. At operating points farther from the design operating point, the measured figure of merit is much lower, as streaming-driven convection adds a third loss mechanism.

\section{Introduction}

Lee, Kittel, Timmerhaus, and Radebaugh ${ }^{2}$ observed a toroidal steady flow in a pulse tube, realized that it must convect significant heat, explained that it might be a streaming phenomenon driven by the sidewall boundary layer, and proposed that tapering the pulse tube might reduce it. Olson and $\mathrm{Swift}^{3}$ developed this idea further, derived an expression for the taper that suppresses streaming, and presented 
measurements showing that this remarkably small taper indeed yields improved pulsetube-refrigerator performance.

Here, we present further experimental evidence that streaming driven convection is suppressed in a pulse tube tapered according to the formula of Olson and Swift. Our measurements show that, with the correct taper, the heat load on the cold heat exchanger due to the pulse tube can be close to that due to ordinary conduction in the pulse tube wall plus ordinary thermoacoustic heat transport near the pulse tube wall.

\section{Apparatus and Instrumentation}

The pulse tube refrigerator used for these measurements, shown schematically in Fig. 1, was part of Cryenco's 1997 thermoacoustically driven orifice pulse tube refrigerator, which liquefied natural gas at rates as high as 100 gallons per day. ${ }^{4,5}$ This refrigerator is much more powerful than those usually discussed at the International Cryocooler Conferences; its measured net cooling power was between $1100 \mathrm{~W}$ and $1700 \mathrm{~W}$ at $125 \mathrm{Kelvin}$ for the data reported here. This high power was achieved primarily through use of large-diameter components; for example, the pulse tube itself had a diameter of $10 \mathrm{~cm}$. The refrigerator used $3.1 \mathrm{MPa}$ helium at $40 \mathrm{~Hz}$; for all measurements reported here, the pressure amplitude was $210 \mathrm{kPa}$ at the pressure sensor near the hot end of the pulse tube (see Fig. 1).

In a pulse tube refrigerator with such a large cooling power, the use of inertance ${ }^{6,7}$ is easier than in smaller systems. A large inertance has relatively little dissipation, so series and parallel orifice valves, as shown in Fig. 1, can be used with a large inertance to allow adjustments of the refrigerator's operating point over a wide range. ${ }^{8}$ In this 
paper, we will identify refrigerator operating points by means of the complex acoustic impedance $Z$ at the hot end of the pulse tube, as shown in Fig. 1, just below the middle flow straightener. The magnitude of $Z$ is the ratio of oscillatory pressure amplitude to oscillatory volumetric velocity amplitude; the phase of $Z$ is the phase angle by which pressure leads volumetric velocity.

In the design of our refrigerator, calculations ${ }^{9,10}$ of viscous and thermal losses in the regenerator led us to identify an operating point with the highest expected efficiency at the desired cooling power, which we will call the "design" operating point and which is shown as the open circle in Fig. 2. Analysis using Eq. (13) of Olson and Swift showed that operating points along the line marked "straight" in Fig. 2 would have zero streaming-driven convection if a straight pulse tube were built. We judged that the design operating point was too far from this line, so our pulse tube was built using Eq. (13) for the design operating point, with a taper $(1 / A) d A / d x=-0.49 \mathrm{~m}^{-1}$, which is equivalent to a total included angle $\phi$ of $1.3^{\circ}$ with the cold end larger than the hot end. Further analysis using Eq. (13) showed that all operating points on the line marked "as built" would have zero streamingdriven convection with this taper. The five selected experimental operating points, shown as filled circles and labeled " 1 " through " 5 ," were chosen to be close to the design operating point and close to the "as-built" line of possible operating points. We predicted that experimental operating points farthest from this line would exhibit the greatest streaming-driven convective heat transfer.

To detect the presence of this convective heat transfer, we measured the total energy flow $\dot{H}$ up the pulse tube and the acoustic (or $p V$ ) power $\dot{W}$ up the pulse tube. In the absence of any heat transfer in the pulse tube (due to conduction, 
convection, or radiation), $\dot{H}=\dot{W}$, so the deviation of $\dot{H} / \dot{W}$ from unity is a measure of such undesired heat transfer, which directly reduces the net cooling power of the refrigerator. Hence, this ratio is commonly known as the figure of merit for a pulse tube. In most previous pulse tube refrigerators, the pulse tube figure of merit has typically been in the range from 0.6 to 0.85 . For our experiments, all parts of the pulse-tube refrigerator (including valves, inertance, and compliance) were thermally insulated, except for the fluid streams in contact with the heat exchangers. Hence, measurement of the heat carried away from the heat exchanger at the hot end of the pulse tube was a direct measurement of $\dot{H}$. Uncertainty in our measurements of $\dot{H}$ was about $1 \%$ [check DVM specs.], due mostly to uncertainty in the measurement of the electric current in a heater in the water stream, which was essentially used to determine the water flow rate. [Greg find ref to this technique]. Pressure sensors in the compliance and near the hot end of the pulse tube allowed measurement of acoustic power $\dot{W}$ up the pulse tube. If the void volumes in the hot heat exchanger, inertance, orifice, valves, and associated plumbing were zero, $\dot{W}$ and $Z$ could be obtained from such pressure measurements in a straightforward way ${ }^{8}$ known to most pulse-tube experimentalists. However, in our hardware these void volumes were not negligible, so we used a complete numerical model ${ }^{9}$ containing the geometry of these components, with the measured pressure amplitudes and phases, to obtain $\dot{W}$ and $Z$. This more sophisticated method yielded values of $\dot{W}$ and $Z 5 \%$ to $10 \%$ different from those which would have resulted from the assumption of zero void volumes. The uncertainties in these values of $\dot{W}$ and $Z$ were about $2 \%$, due mostly to uncertainty in the compliance volume. [Greg check w Mark.] 


\section{Results}

Figure 3 shows the experimental values of $\dot{H} / \dot{W}$ for the five experimental operating points, displayed vs how far those operating points are from operating points of predicted zero streaming-driven convection shown as the "as built" line in Fig. 2. To provide a quantitative measure of this distance, the horizontal axis is the difference between the "as-built" value of $(1 / A) d A / d x$ and the value that Eq. (13) yields for $(1 / A) d A / d x$ at the selected operating point. Operating points 2 and 5 , which were closest to the "as built" optimal condition, have the highest value of $\dot{H} / \dot{W}$, approximately 0.96. Based on the lower values of $\dot{H} / \dot{W}$ for the other three operating points, we estimate that operating points 2 and 5 would have yielded an experimental value of $\dot{H} / \dot{W}$ near or below 0.85 if the pulse tube had been straight instead of being tapered according to Eq. (13).

The solid line in Fig. 3 near $\dot{H} / \dot{W}=0.97$ shows calculated values of $\dot{H} / \dot{W}$ including only thermal conductivity in the stainless-steel pulse tube wall and thermoacoustic boundary-layer heat transport, but not including streaming-driven convection. (As in Fig. 2, the design operating point is indicated by the open circle.) Hence, within our experimental uncertainties of a few percent in $\dot{H} / \dot{W}$, experimental operating points 2 and 5 exhibit no streaming-driven convection.

The dashed lines in Fig. 3 are rough estimates of $\dot{H} / \dot{W}$ taking streaming-driven connection into account, using Eqs. (10) and (11) of Olson and Swift evaluated at the center (axially) of the pulse tube, and calculating the heat load with the assumption that all the downward streaming gas is at room temperature and all the upward moving gas is at $125 \mathrm{~K}$. These lines are in reasonable agreement with the data. They 
must be regarded as rough estimates because many of the assumptions built into Olson and Swift's work are only valid in the zero-streaming limit.

\section{Summary}

Streaming-driven convection can be eliminated in a pulse tube, either by tapering the pulse tube or by choosing an operating point for which a straight pulse tube generates no streaming. Streaming is eliminated if the pulse tube is tapered according to Eq. (13) of Olson and Swift:

$$
\begin{aligned}
\frac{1}{A}\left(\frac{d A}{d x}\right)= & -\frac{\omega\left|p_{1}\right|}{\gamma p_{m}\left|\left\langle u_{1}\right\rangle\right|}\left[\left(1+\frac{2(\gamma-1)\left(1-b \sigma^{2}\right)}{3 \sigma(1+\sigma)}\right) \cos \theta\right. \\
& \left.+\left(1+\frac{2(\gamma-1)(1-b) \sqrt{\sigma}}{3(1+\sigma)}\right) \sin \theta\right]-\frac{(1-b)(1-\sqrt{\sigma})}{3(1+\sigma)(1+\sqrt{\sigma})} \frac{d T_{m} / d x}{T_{m}} \\
\simeq & -(0.75 \cos \theta+0.64 \sin \theta) \frac{\omega\left|p_{1}\right|}{p_{m}\left|\left\langle u_{1}\right\rangle\right|}-0.0058 \frac{d T_{m} / d x}{T_{m}}
\end{aligned}
$$

If streaming is thus eliminated, the heat flow $\dot{Q}=\dot{H}-\dot{W}$ down the pulse tube can be estimated accurately using

$$
\begin{aligned}
\dot{Q}= & K_{\text {wall }} A_{\text {wall }} \frac{d T_{m}}{d x}+K A \frac{d T_{m}}{d x} \\
& +\frac{\Pi \delta_{\kappa}}{4}\left[\left(\frac{1-\sqrt{\sigma}}{1+\sigma} \cos \theta-\frac{1+\sqrt{\sigma}}{1+\sigma} \sin \theta\right)\left|p_{1}\right|\left|\left\langle u_{1}\right\rangle\right|+\frac{1-\sigma^{3 / 2}}{1-\sigma^{2}} \frac{d T_{m}}{d x} \frac{\rho_{m} c_{p}\left|\left\langle u_{1}\right\rangle\right|^{2}}{\omega}\right] \\
\simeq & K_{\text {wall }} A_{\text {wall }} \frac{d T_{m}}{d x}+K A \frac{d T_{m}}{d x} \\
& +\Pi \delta_{\kappa}\left[(0.025 \cos \theta-0.27 \sin \theta)\left|p_{1}\right|\left|\left\langle u_{1}\right\rangle\right|+0.51 \frac{d T_{m} / d x}{T_{m}} \frac{p_{m}\left|\left\langle u_{1}\right\rangle\right|^{2}}{\omega}\right]
\end{aligned}
$$

Here, $A$ is the internal area of the pulse tube and $A_{\text {wall }}$ is the cross-sectional area of its wall, $\Pi$ is the internal perimeter of the pulse tube, $x$ is the axial distance from the 
cold end of the pulse tube, $\omega$ is the angular frequency of oscillation, $T_{m}$ is the mean temperature, $p_{m}$ is the mean pressure, $\left|p_{1}\right|$ is the amplitude of the oscillatory pressure, $\left|\left\langle u_{1}\right\rangle\right|$ is the amplitude of the lateral spatial average of the oscillatory velocity, $\theta$ is the phase angle by which $\left\langle u_{1}\right\rangle$ leads $p_{1}, \rho_{m}$ is the mean density of the gas, $c_{p}$ is its isobaric heat capacity per unit mass, $\gamma$ is its ratio of isobaric to isochoric specific heats, $K$ is its thermal conductivity, $\sigma$ is its Prandtl number, $b=\left(T_{m} / \mu_{m}\right)\left(d \mu_{m} / d T_{m}\right)$ where $\mu_{m}$ is its dynamic viscosity, $\delta_{\kappa}=\sqrt{2 K / \omega \rho c_{p}}$ is its thermal penetration depth, and $K_{\text {wall }}$ is the thermal conductivity of the wall material. The second form of each equation incorporates numerical values for low-temperature helium gas: $\gamma=5 / 3, \sigma \simeq 0.69$, and $b \simeq 0.68$. In Eqs. (3) and (4), the first two terms account for ordinary heat conduction, and the last two terms are from Rott's second-order ("thermoacoustic") energy flux ${ }^{11}$ in the limit $R \gg \delta_{\kappa} \cdot{ }^{12}$ (Note that pulse tube end effects can cause $d T_{m} / d x$ to be significantly larger than $\Delta T / \Delta x$, where $\Delta T$ is the temperature difference between the two heat exchangers and $\Delta x$ is the length of the pulse tube.) In a large pulse tube, a figure of merit $\dot{H} / \dot{W}>0.95$ is possible, as demonstrated here. In smaller pulse tubes, for which boundary-larger thermoacoustic processes carry a larger fraction of the heat, figures of merit $>0.9$ should be possible.

Careful attention to detail is required to achieve such performance. The operating point $Z$ of the refrigerator must be planned very accurately, or means to finely adjust it must be provided. Flow straightening at the ends of the pulse tube must eliminate jet-driven convection. The pulse tube wall roughness must be much less that the viscous penetration depth, and of course the pulse tube must operate in the laminar or weakly turbulent regime. 


\section{Acknowledgments}

This work has been supported by Cryenco, Inc. and by the Office of Fossil Energy in the US Department of Energy. 


\section{Research Bibliography}

${ }^{1}$ Permanent address: Condensed Matter and Thermal Physics Group, Mail Stop K764, Los Alamos National Laboratory, Los Alamos NM 87545.

${ }^{2}$ J. M. Lee, P. Kittel, K. D. Timmerhaus, and R. Radebaugh. Flow patterns intrinsic to the pulse tube refrigerator. In Proceedings of the 7th International Cryocooler Conference, page 125, Kirtland AFB, NM 87117-5776, 1993. Phillips Laboratory.

${ }^{3}$ J. R. Olson and G. W. Swift. Acoustic streaming in pulse tube refrigerators: Tapered pulse tubes. Cryogenics, ??:??, 1997.

${ }^{4}$ J. J. Wollan. dont know yet. 1997. the proceedings for your European trip.

${ }^{5}$ G. W. Swift. Thermoacoustic natural gas liquefier. 1997. In proceedings of the DOE Natural Gas Conference, Houston TX, March 1997.

${ }^{6}$ S. W. Zhu, S. L. Zhou, N. Yoshimura, and Y. Matsubara. Phase shift effect of the long neck tube for the pulse tube refrigerator. In To Be Published in the Proceedings of Cryocoolers 9, Held June 1996 in New Hampshire, 1996.

${ }^{7} \mathrm{R}$. Radebaugh. Advances in cryocoolers. 1996. To be published in the proceedings of the 1996 International Cryogenic Engineering Conference (Kitakyushu, Japan, May 1996).

${ }^{8}$ D. L. Gardner and G. W. Swift. Use of inertance in orifice pulse tube refrigerators. Cryogenics, 37:117-121, 1997.

${ }^{9}$ W. C. Ward and G. W. Swift. Design environment for low amplitude thermoacoustic engines (DeltaE). J. Acoust. Soc. Am., 95:3671-3672, 1994. Fully tested software 
and users guide available from Energy Science and Technology Software Center, US Department of Energy, Oak Ridge, Tennessee. To review DeltaE's capabilities, visit the Los Alamos thermoacoustics web site at http://rott.esa.lanl.gov/. For a beta-test version, contact ww@lanl.gov (Bill Ward) via Internet.

${ }^{10}$ G. W. Swift and W. C. Ward. Simple harmonic analysis of regenerators. Journal of Thermophysics and Heat Transfer, 10:652-662, 1996.

${ }^{11} \mathrm{~N}$. Rott. Thermally driven acoustic oscillations, part iii: Second-order heat flux. $Z$. Angew. Math. Phys., 26:43, 1975.

${ }^{12}$ G. W. Swift. Encyclopedia of Applied Physics, volume 21, chapter Thermoacoustic engines and refrigerators, pages 245-264. Wiley, for American Institute of Physics, 1997.

\section{Figure captions}

Fig. 1 Schematic of the apparatus. "P" indicates locations of pressure sensors. The angle $\phi$ of the tapered pulse tube has been greatly exaggerated.

Fig. 2 Complex acoustic impedance $Z$ at the hot end of the pulse tube. The open circle is the design operating point, and the line through the point indicates $Z$ 's for which the as-built taper should suppress streaming. The dashed line indicates $Z$ 's which would have had zero streaming if the pulse tube had been built straight. The filled circles indicate the experimental operating points.

Fig. 3 Figure of merit of the pulse tube at various operating points vs how far each operating point is from the "as-built" line on Fig. 2. Filled circles show experimental values. Open circle shows the design operating point; solid line shows 
calculated $\dot{H} / \dot{W}$ neglecting streaming; dashed line shows rough calculated estimate of $\dot{H} / \dot{W}$ including streaming. 


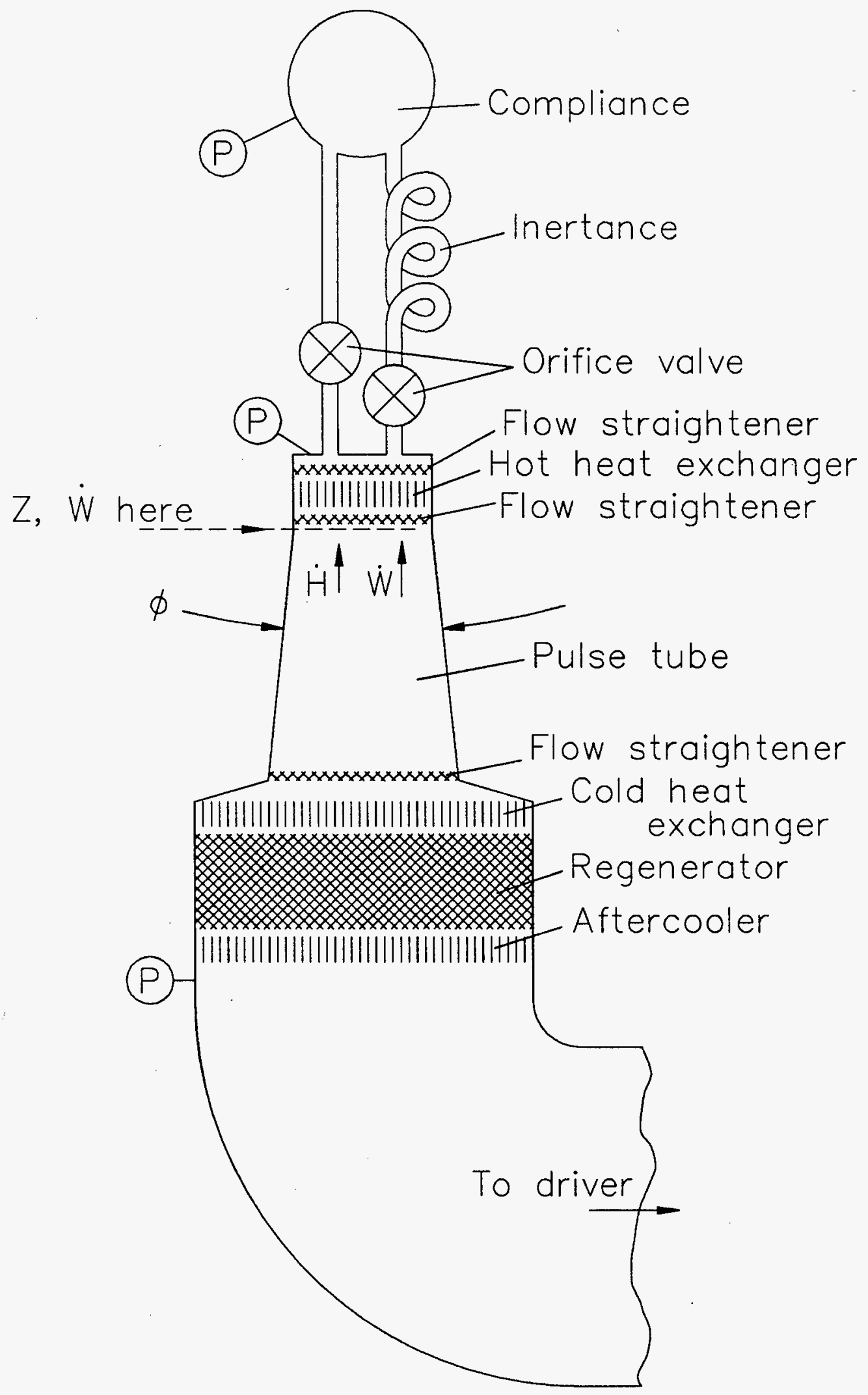

Fig. One, 
6

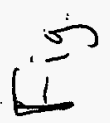

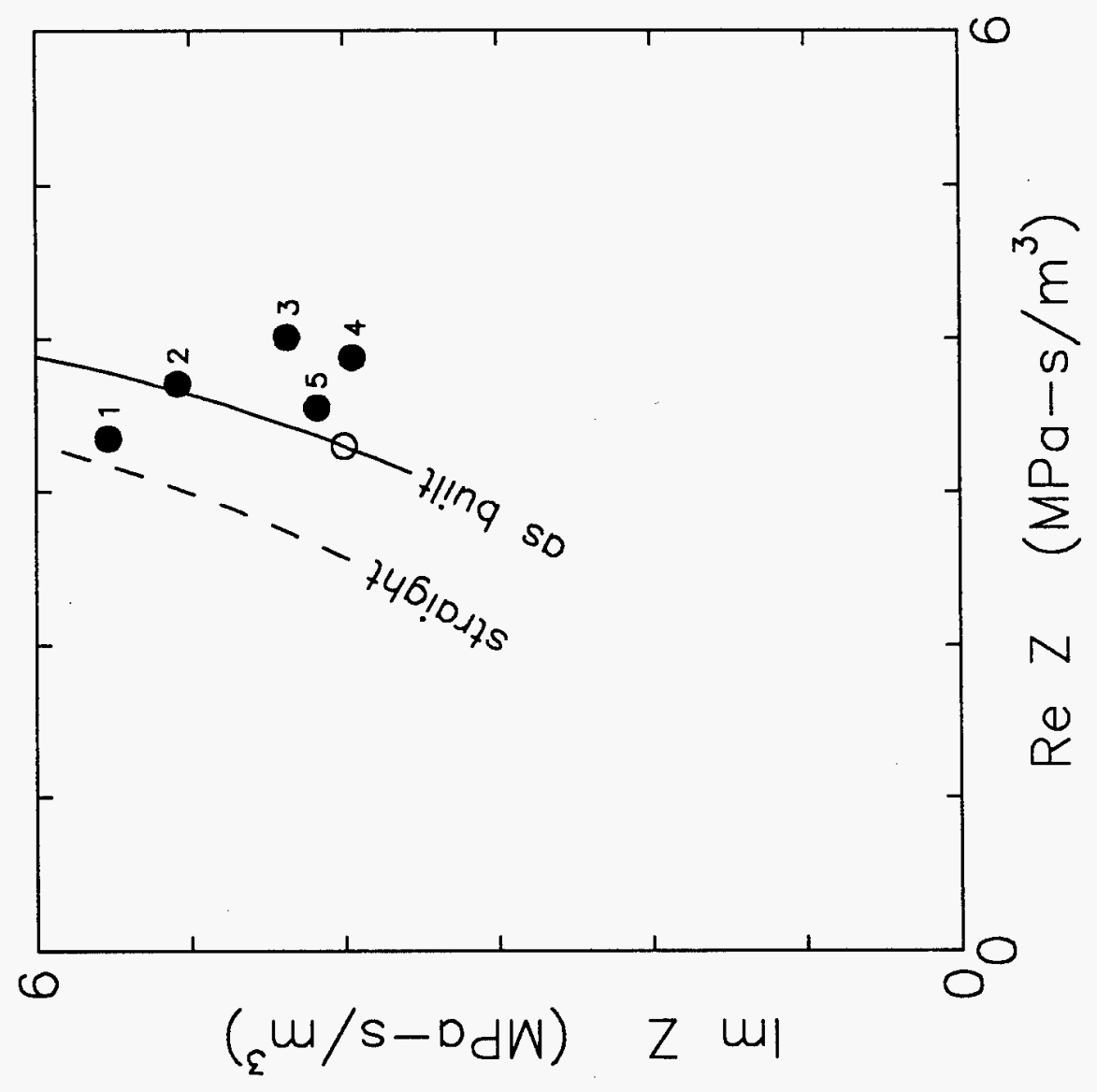




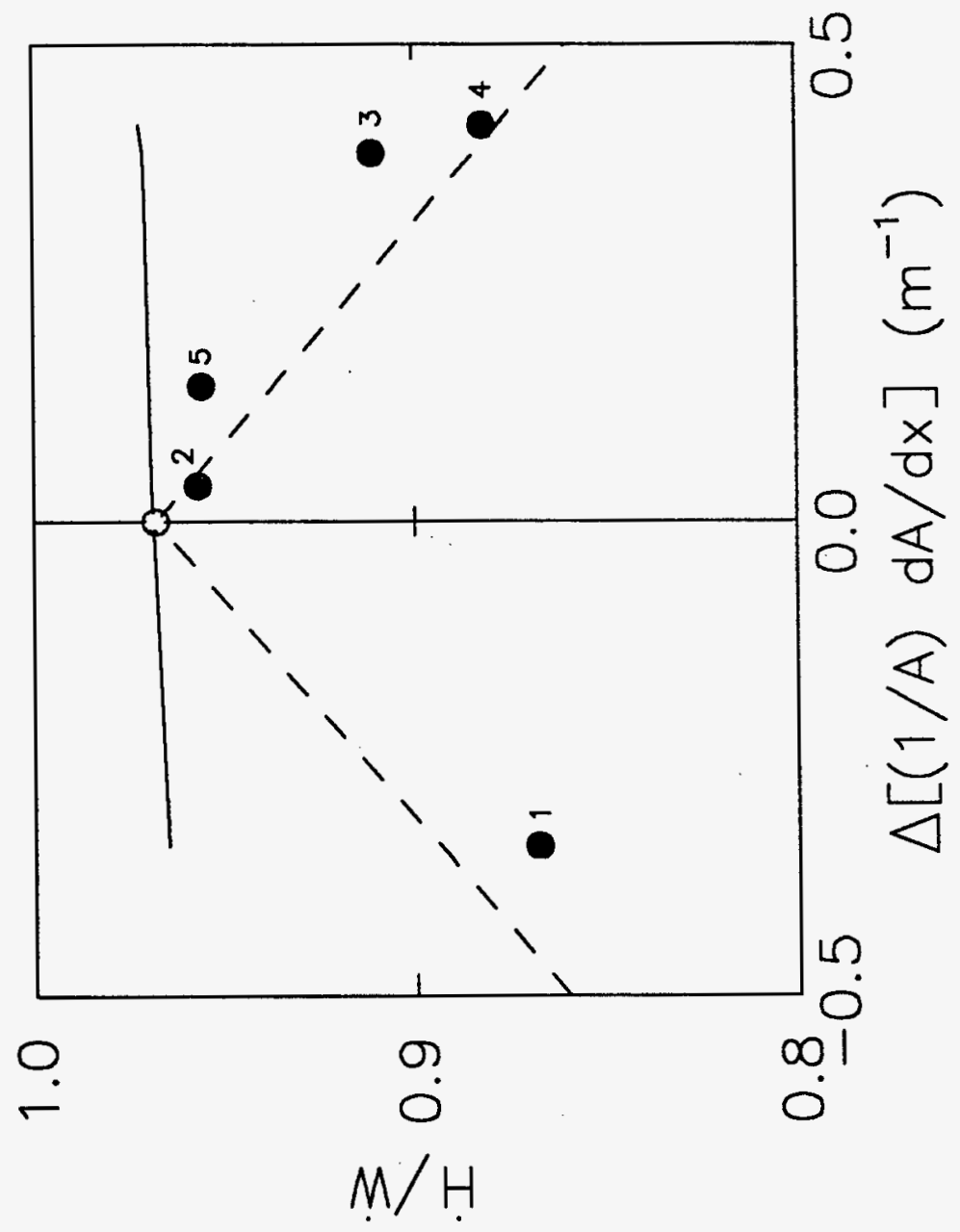




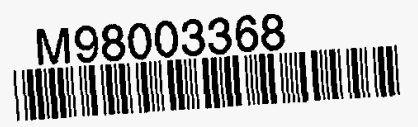

Report Number (14)LA-VR--97-4713

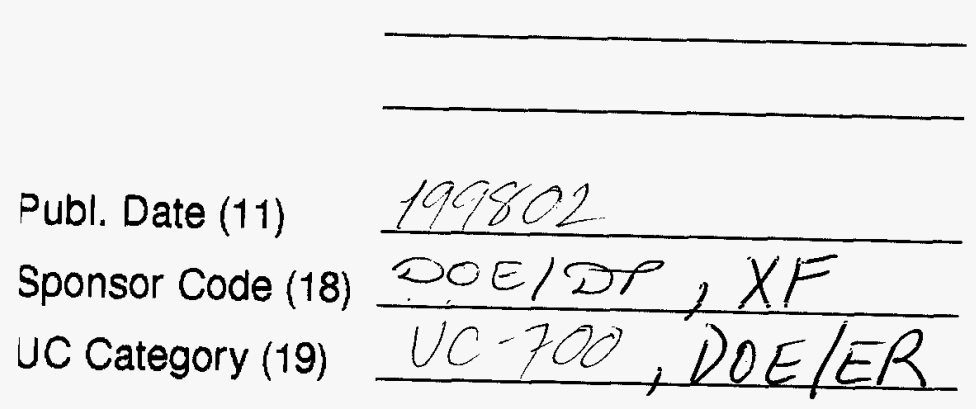

